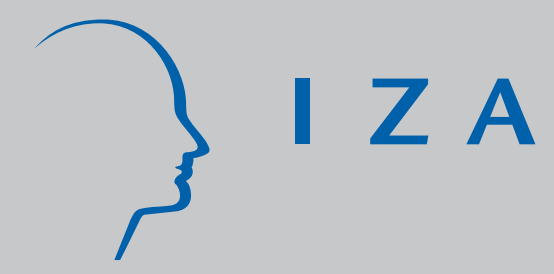

IZA DP No. 2059

Doping and Cheating in Contest-Like Situations

Matthias Kräkel

March 2006 


\title{
Doping and Cheating in Contest-Like Situations
}

\author{
Matthias Kräkel \\ University of Bonn and IZA Bonn
}

Discussion Paper No. 2059

March 2006

\author{
IZA \\ P.O. Box 7240 \\ 53072 Bonn \\ Germany \\ Phone: +49-228-3894-0 \\ Fax: +49-228-3894-180 \\ Email: iza@iza.org
}

\begin{abstract}
Any opinions expressed here are those of the author(s) and not those of the institute. Research disseminated by IZA may include views on policy, but the institute itself takes no institutional policy positions.

The Institute for the Study of Labor (IZA) in Bonn is a local and virtual international research center and a place of communication between science, politics and business. IZA is an independent nonprofit company supported by Deutsche Post World Net. The center is associated with the University of Bonn and offers a stimulating research environment through its research networks, research support, and visitors and doctoral programs. IZA engages in (i) original and internationally competitive research in all fields of labor economics, (ii) development of policy concepts, and (iii) dissemination of research results and concepts to the interested public.
\end{abstract}

IZA Discussion Papers often represent preliminary work and are circulated to encourage discussion. Citation of such a paper should account for its provisional character. A revised version may be available directly from the author. 
IZA Discussion Paper No. 2059

March 2006

\section{ABSTRACT}

\section{Doping and Cheating in Contest-Like Situations ${ }^{*}$}

Individuals who compete in a contest-like situation (for example, in sports, in promotion tournaments, or in an appointment contest) may have an incentive to illegally utilize resources in order to improve their relative positions. We analyze such doping or cheating within a tournament game between two heterogeneous players. Three major effects are identified which determine a player's doping decision - a cost effect, a likelihood effect and a windfall-profit effect. Moreover, we discuss whether the favorite or the underdog is more likely to be doped, the impact of doping on overall performance, the influence of increased heterogeneity on doping, the welfare implications of doping, and possible prevention of doping.

JEL Classification: J3, K42, M5

Keywords: cheating, contest, doping, fraud in research, tournament

Corresponding author:

Matthias Kräkel

BWL II

University of Bonn

Adenauerallee 24-42

D-53113 Bonn

Germany

Email: m.kraekel@uni-bonn.de

\footnotetext{
* I would like to thank Marion Eberlein, Christian Grund, Oliver Gürtler, Judith Przemeck, Patrick Schmitz and two anonymous referees for very helpful comments. Financial support by the Deutsche Forschungsgemeinschaft (DFG), grants KR 2077/2-3 and SFB/TR 15 "Governance and the Efficiency of Economic Systems", is gratefully acknowledged.
} 


\section{Introduction}

In practice, there exist diverse competitive situations in which individuals illegally utilize resources in order to improve their positions. Such behavior can be characterized as doping or cheating. Naturally, we associate doping with professional sports where athletes sometimes take drugs to improve their performance so that their probability of winning a contest increases. Perhaps most spectacular are the cases of detected doping along with professional cycling in the last decade - in particular the disqualification of the Festina athletes during the Tour de France 1998. Moreover, there are also several well-known cases of revealed doping in connection with the Olympic Games.

However, doping or cheating as defined above can also take place in other contexts. For example, we can imagine that employees try to bribe customers or supervisors within a hierarchical contest in order to win promotion to a higher level. The first example - bribing of customers - can also be called corruption: ${ }^{1}$ We can think, for example, of a sub-supplier's salesman who bribes an employee of another firm so that this firm orders the salesman's initial products. The bribing of supervisors has been discussed in the context of influence activities in the literature. ${ }^{2}$ In this case, the employee transfers a monetary or non-monetary side payment to his supervisor in order to get excellent marks which improve his promotion chances. In addition, fraudulent accounting to embellish the financial status of a firm represents another example of doping in business. ${ }^{3}$

Furthermore, we can remember doping or cheating cases in which scientists manipulated research results in order to improve their reputations. Improved reputation then serves to enhance one's chances of getting additional

\footnotetext{
${ }^{1}$ For the economics of corruption see, for example, Tirole (1996).

${ }^{2}$ See Fairburn and Malcomson (1994).

${ }^{3}$ See Berentsen and Lengwiler (2004).
} 
research funds or attractive positions either at universities or in industry. In particular, the perils of cheating may be highest in experimental research where cheating can hardly be detected. The importance of scientific fraud are indicated exemplarily by several US reports on cheating and fraud in science. ${ }^{4}$ Moreover, Martinson et al. (2005) surveyed several thousand scientists in the US and found that $33 \%$ of the respondents have engaged in fraudulent research in the previous three years. Whereas only $0.3 \%$ of the scientists have directly falsified their research data, $6.0 \%$ have failed to present data that contradict their own previous research, and $15.5 \%$ have changed the design, methodology or results of a study in response to pressure from a funding source. Of course, the estimates of Martinson et al. should be very conservative as misbehaving scientists ought to be less likely to give correct answers about fraudulent behavior. The most recent case of spectacular cheating in science comes from South Korea. In December 2005, the world-renowned stem-cell researcher Hwang Woo-suk of Seoul National University resigned his post after investigators found that he had faked pioneering results on cloning.

Finally, note that our discussion of doping and cheating in contest-like situations nearly applies to any situation in which individuals are evaluated relative to other persons or relative to certain standards. In the latter case, the rationally acting opponent in our formal model only has to be replaced with an exogenously given standard a player has to beat. Basically, in each situation in which stress of performance is sufficiently high any individual

\footnotetext{
4"Fraud and Misrepresentation in Science" (Report F. I-88) of the Council on Scientific Affairs, joint report "Scientific Fraud and Misrepresentation" (Report I-89) of the Council on Ethical and Judical Affairs and the Council on Scientific Affairs, "Framework for Institutional Policies and Procedures to Deal with Fraud in Research" of the Association of American Universities, the National Association of State Universities and Land Grant Colleges, and the Council on Graduate Schools from the years 1988 and 1989.
} 
has to decide whether to keep the rules of the game or not. If not, illegal or manipulating behavior is used to gain an individual advantage which can be summarized as doping or cheating. Hence, also the cheating of students in exams belongs to this wide class misbehavior.

Although these examples are very different, they all have the common characteristics of doping or cheating as defined above: There is a tournament or contest-like situation between individuals who compete for a given winner prize (e.g. a medal, a monetary prize, research funds, promotion or appointment to an attractive position), and these individuals have the opportunity to increase their winning probabilities by using illegal activities. Of course, if such behavior is detected, the respective individual will be excluded from the competition, i.e. he will get defaulted. These heterogeneous examples should demonstrate that the doping problem is a relevant topic not only in professional sports but also in a lot of other contest-like situations.

The economic consequences of doping are meaningful. For example, if doping instead of ability and/or effort is decisive for promotion and appointment decisions, there will be a misallocation of talent and/or a decrease in incentives given that competitors observe the impact of doping. Furthermore, doping is meaningful from an economic perspective because lots of resources are spent for the implementation of drug tests in order to prevent doping in professional sports.

This paper concentrates on the doping game between two players of different ability in order to address the following questions: Are favorites or underdogs more likely to be doped? Does doping increase or decrease overall performance? Does increased heterogeneity increase or decrease the likelihood of doping? Is mutual doping welfare enhancing or decreasing? What policies should be adopted in the latter case? Are drug tests prior (ex-ante 
testing) or after the tournament (ex-post testing) preferable? Note that we focus on the game between the two players who have to decide on both doping and the use of legal inputs (i.e., effort, training or - more general - investment). In the discussion, we will point to several possibilities which can be used to prevent doping. However, we do not explicitly solve the optimization problem of a contest organizer who has to decide on the design of the contest. One important reason for this restriction is that from an economic perspective it is not clear whether the contest organizer (e.g. a private investor, a sport league or society) really wants to prevent doping.

We identify three effects which determine the use of drugs in tournaments. The first effect is called likelihood effect which covers the aspect that taking drugs enhances one's own likelihood of winning given that doping is not detected. The second effect is labelled cost effect: Recall that in the model we assume that the contestants also invest in legal inputs to win the tournament. Depending on the impact of doping on the outcome of the tournament and depending on other parameters, doping may or may not increase overall investment incentives and, therefore, also investment costs. The third effect is named windfall-profit effect: If one player is got defaulted because of doping and the other player not, the latter one will receive the winner prize for sure without having outperformed his competitor. The interplay of these three effects determines whether mutual doping is an equilibrium. For a certain kind of welfare function it is shown that mutual doping increases welfare under socially optimal tournament prizes. If the organizer of the tournament wants to prevent doping, he should choose a large loser prize and a small spread between winner and loser prize. The results also indicate that, under reasonable assumptions, the favorite is more likely to be doped than the underdog. However, if expected costs from getting defaulted (e.g. loss of 
reputation) are very high for favorites relative to underdogs, the former ones will tend not to be doped.

There exist two strands of related literature. First, there are some papers on doping and cheating in sporting contests. Eber and Thépot (1999) consider two homogeneous athletes who have to choose between doping $(D)$ and no-doping $(N D)$, but do not exert legal inputs such as effort or investment. Depending on the parameter constellations, each of the four combinations $(D, D),(N D, N D),(D, N D)$, and $(N D, D)$ can be an equilibrium. Moreover, by doing comparative statics the two authors analyze several possibilities to prevent doping. However, they do not discuss why society should deter athletes from taking drugs. Maennig (2002) does not consider a technical model on doping. Instead he points to the parallels between doping and corruption in international sports. As a measure against doping, he suggests high financial penalties for the athletes who got defaulted due to a positive doping test. Of course, this solution will only work, if individuals do not face problems of limited liability. Haugen (2004) uses a simple game-theoretic model in order to discuss doping of two homogeneous athletes who do not choose efforts or investments. Under certain assumption he obtains an equilibrium in which both players takes drugs. Since doping implies a disutility when being caught, this equilibrium is a kind of prisoner's dilemma, i.e. both would be better off with not taking drugs. ${ }^{5}$ Berentsen (2002) considers doping of heterogeneous players in a contest model. Again, input decisions on effort or investments are neglected. Berentsen shows that, for certain parameter constellations, a mixed-strategy equilibrium exists in which the favorite has a higher probability of taking drugs than the underdog. However, the favorite does not always have a higher probability of winning. Preston and

\footnotetext{
${ }^{5}$ Bird and Wagner (1997) argue in a similar way that doping leads to a prisoner'sdilemma like situation.
} 
Szymanski (2003) focus on different forms of cheating in contests - doping, sabotage, and match fixing. They discuss how these forms of cheating arise and how society can deal with them. Konrad (2005), contrary to the papers before but in line with this paper, discusses a tournament model in which players choose both doping and legal inputs. However, contrary to this paper players are homogeneous, and the probability of getting defaulted is zero. Konrad shows that, given a welfare maximizing winner prize, mutual doping is always welfare enhancing. Finally, Berentsen et al. (2004) discuss cheating and doping in an evolutionary game in order to determine those factors which enhance the dissemination of doping within society.

The second strand of literature deals with sabotage in tournaments or contests. Similar to doping, a player gains a relative competitive advantage by choosing sabotage. However, this relative advantage arises from decreasing a competitor's output and not by illegally increasing one's own output. Papers that deal with this subject are Lazear (1989), Konrad (2000), Chen (2003) and Kräkel (2005). Contrary to the doping literature, sabotaging players never get defaulted but have to bear costs of exerting sabotage.

The paper is organized as follows: In the next section, a tournament model with doping is introduced. This model is analyzed in Section 3 which also contains the main results. Additional results are offered in Section 4, in which ex-ante testing is compared to ex-post testing, and both endogenous tournament prizes and the implications of doping on welfare are discussed. The paper concludes in Section 5.

Note that although the problem of using illegal behavior in order to gain a competitive advantage is applicable to very different situations, in the following most of the examples will come from sports. 


\section{The Model}

We consider a rank-order tournament between two risk neutral, heterogeneous players or athletes $U$ and $F{ }^{6}$ Here, $U$ indicates the less able player (the underdog) and $F$ the more able one (the favorite). The output or performance of player $i(i=U, F)$ can be described by the function ${ }^{7}$

$$
q_{i}=d_{i} t_{i} \mu_{i}+\varepsilon_{i}
$$

$\mu_{i}$ denotes the legal input of player $i$ which is endogenously chosen by him for improving his performance. This input may be effort or training, for example. According to Lazear and Rosen (1981, p. 842) we will refer to this variable more generally as investment. $t_{i}$ describes player $i$ 's exogenously given talent or ability with $t_{U}=1$ denoting underdog $U$ 's talent, and $t_{F}=$ $\tau>1$ the talent of favorite $F$. The variable $d_{i}$ describes the illegal input or doping decision of player $i$. Each player can only choose between two values $d_{i}=1$ (no-doping) and $d_{i}=d>1$ (doping). ${ }^{8}$ While abilities $t_{U}$ and $t_{F}$ are assumed to be common knowledge, each player cannot observe the doping decision of his opponent. $\varepsilon_{i}(i=U, F)$ is an exogenously given random term. It stands for luck, noise or measurement error. $\varepsilon_{U}$ and $\varepsilon_{F}$ are assumed to be identically and independently distributed (i.i.d.). Let $F(\cdot)$ denote the cumulative distribution function (cdf) of the composed random variable $\varepsilon_{j}-\varepsilon_{i}(i, j=U, F ; i \neq j)$, being continuously differentiable, and $f(\cdot)$ the corresponding density. We assume that $f(\cdot)$ has a unique mode at

\footnotetext{
${ }^{6}$ Most of the assumptions follow the seminal tournament paper by Lazear and Rosen (1981).

${ }^{7}$ As in Konrad (2005), both inputs are complements. See also Epstein and Hefeker (2003) on a contest model with two inputs which are complementary.

${ }^{8}$ The simple choice between doping and no-doping sketches the idea that, if a player decides to take drugs, he will choose the optimal degree of doping that solves the trade-off between effectiveness and the probability of being caught.
} 
zero. ${ }^{9}$ Note that, due to the i.i.d.-assumption, the density $f(\cdot)$ is symmetric around zero.

It is assumed that doping entails direct costs on player $i$ (e.g. expenditures for drugs or affected health) given by

$$
\kappa_{i}=\left\{\begin{array}{ccc}
\bar{\kappa}_{i}>0 & \text { if } & d_{i}=d \\
0 & \text { if } & d_{i}=1
\end{array}\right.
$$

Investment $\mu_{i}$ also has some costs for player $i$ which are described by $c\left(\mu_{i}\right)$ with $c(0)=0, c^{\prime}\left(\mu_{i}\right)>0$ and $c^{\prime \prime}\left(\mu_{i}\right)>0$ for $\mu_{i}>0$. Depending on the meaning of $\mu_{i}$, costs may be the disutility of effort in monetary terms or the opportunity costs for time-consuming training, for example.

The two players compete for tournament prizes $w_{1}$ and $w_{2}$ with $w_{1}>$ $w_{2}>0$. Let the spread between winner and loser prize be denoted by $\Delta w:=$ $w_{1}-w_{2}$. The prizes are exogenously given. If no player gets defaulted and $q_{i}>q_{j}(i, j=U, F ; i \neq j)$, player $i$ will be declared the contest winner and receives the high winner prize $w_{1}$, whereas player $j$ only receives the loser prize $w_{2}$. If one player is accused for being doped and gets defaulted, he will receive no prize whereas the other player gets the winner prize $w_{1}$. Let the probability of not getting defaulted (or non-detection probability) be $\delta_{i}=\delta \in(0,1)$ if the player has chosen $d_{i}=d$, and $\delta_{i}=1$ if he has chosen $d_{i}=1$. In other words, only if a player has decided to take drugs, there will be a positive probability of being accused and getting defaulted. ${ }^{10}$ Finally,

\footnotetext{
${ }^{9}$ For example, $\varepsilon_{j}$ and $\varepsilon_{i}$ may be normally distributed with mean $m$ and variance $\sigma^{2}$. Then the convolution $f(\cdot)$ again describes a normal distribution with $\varepsilon_{j}-\varepsilon_{i} \sim N\left(0,2 \sigma^{2}\right)$. If $\varepsilon_{j}$ and $\varepsilon_{i}$ are uniformly distributed, the distribution of $\varepsilon_{j}-\varepsilon_{i}$ will be triangular with mean zero. In addition, the assumption is not unusual in the tournament literature; see, e.g., Drago et al. (1996), Chen (2003).

${ }^{10}$ Berentsen (2002) considers the possible case of a wrong test result for a player who has decided not to take drugs. This case may happen if, for example, the doping test is imperfect or the player has consumed an illegal drug unintentionally. Note that our results
} 
we assume that doping also implies indirect costs for a player since he faces a loss $L_{i}$ (e.g. loss of reputation or an income loss due to suspension in future contests) if getting defaulted (with probability $1-\delta_{i}$ ).

In order to give explicit solutions in Corollaries 1 and 2, at the end of Subsection 3.2 we will use parametric specifications for the cost function $c(\cdot)$ and the convolution $f(\cdot)$. Costs for legal investments are assumed to be quadratic with $c\left(\mu_{i}\right)=\frac{c}{2} \mu_{i}^{2}$ and $c>0 . \quad \varepsilon_{U}$ and $\varepsilon_{F}$ are assumed to be uniformly distributed over $[-\bar{\varepsilon}, \bar{\varepsilon}]$ so that the composed random variable $\varepsilon_{j}-\varepsilon_{i}$ is described by a triangular distribution ${ }^{11}$ over $[-\gamma, \gamma]:=\left[-\frac{1}{2 \bar{\varepsilon}}, \frac{1}{2 \bar{\varepsilon}}\right]$ with density

$$
f(x)=\left\{\begin{array}{ccc}
\gamma+\gamma^{2} x & \text { if } & -\frac{1}{\gamma} \leq x \leq 0 \\
\gamma-\gamma^{2} x & \text { if } & 0<x \leq \frac{1}{\gamma} \\
0 & & \text { otherwise }
\end{array}\right.
$$

and corresponding cdf

$$
F(x)=\left\{\begin{array}{cc}
0 & \text { if } x<-\frac{1}{\gamma} \\
\gamma x+\frac{\gamma^{2}}{2} x^{2}+\frac{1}{2} & \text { if }-\frac{1}{\gamma} \leq x \leq 0 \\
\gamma x-\frac{\gamma^{2}}{2} x^{2}+\frac{1}{2} & \text { if } 0<x \leq \frac{1}{\gamma} \\
1 & \text { if } x>\frac{1}{\gamma}
\end{array}\right.
$$

To guarantee concavity of the agents' objective functions with respect to investment choice given that at least one agent is doped, the technical assumptions

$$
c>d^{2} \tau^{2} \Delta w \gamma^{2} \delta \quad \text { and } \quad d^{2} \delta>1
$$

are introduced. ${ }^{12}$ The second inequality allows that it may be attractive for a will remain qualitatively the same, if we assume a positive probability of getting defaulted when not being doped.

${ }^{11}$ For computation of the triangular convolution see, for example, Kräkel (2000).

${ }^{12}$ Calculating the second-order conditions for the two players concerning optimal invest- 
player to be doped, because the impact of doping relative to the non-detection probability is not too low.

The timing of the game is the following: At the first stage, both players simultaneously decide on $d_{i}(i=U, F)$. At the second stage, each player $i$ only knows his own doping decision and chooses his input variable $\mu_{i}(i=U, F)$. After that, nature chooses $\varepsilon_{U}$ and $\varepsilon_{F}$ so that the two players' outputs $q_{U}$ and $q_{F}$ can be compared. Finally, a doping test takes place so that player $i$ gets defaulted with probability $1-\delta_{i}$ depending on his choice of $d_{i}(i=U, F)$.

\section{Results}

In this section, we solve the two-stage game described above. First, we look at the tournament stage where the two players simultaneously choose their legal inputs $\mu_{i}^{*}\left(d_{i}, d_{j}\right)$ and $\mu_{j}^{*}\left(d_{i}, d_{j}\right)$ for given pairs $\left(d_{i}, d_{j}\right)$. Then we consider the first stage where the players decide on their illegal inputs $d_{i}^{*}$ and $d_{j}^{*}$ given the anticipated best responses $\mu_{i}^{*}\left(d_{i}, d_{j}\right)$ and $\mu_{j}^{*}\left(d_{i}, d_{j}\right)$ for the next stage. By assumption, each player cannot observe the doping decision of his opponent. Hence, the game is equivalent to a one-stage game where each player $i$ simultaneously decides on the pair $\left(d_{i}, \mu_{i}\right)$. However, for didactical reasons it makes sense to look at each stage separately.

\subsection{The Tournament Stage}

At the second stage of the game, the two players choose $\mu_{i}$ and $\mu_{j}$, respectively, in order to maximize their expected utilities for given values $d_{i}$ and ment choices by using the triangular distribution described by (2) and (3), shows that the inequality $d_{F}^{2} \tau^{2} \Delta w \gamma^{2} \delta_{U} \delta_{F}<c$ has to be satisfied. 
$d_{j}$. Player $i$ 's probability of winning the tournament can be written as

$$
\begin{aligned}
\operatorname{prob}\left\{q_{i}>q_{j}\right\} & =\operatorname{prob}\left\{d_{i} t_{i} \mu_{i}+\varepsilon_{i}>d_{j} t_{j} \mu_{j}+\varepsilon_{j}\right\} \\
& =F\left(d_{i} t_{i} \mu_{i}-d_{j} t_{j} \mu_{j}\right) .
\end{aligned}
$$

Hence, the underdog's expected utility is given by

$$
\begin{aligned}
E U_{U}\left(\mu_{U} ; \mu_{F}, d_{U}, d_{F}\right)= & \left(w_{2}+\Delta w F\left(d_{U} \mu_{U}-d_{F} \tau \mu_{F}\right)\right) \delta_{U} \delta_{F} \\
& +w_{1} \delta_{U}\left(1-\delta_{F}\right)-L_{U}\left(1-\delta_{U}\right)-\kappa_{U}-c\left(\mu_{U}\right)
\end{aligned}
$$

whereas the favorite wants to maximize

$$
\begin{aligned}
E U_{F}\left(\mu_{F} ; \mu_{U}, d_{U}, d_{F}\right)= & \left(w_{2}+\Delta w\left[1-F\left(d_{U} \mu_{U}-d_{F} \tau \mu_{F}\right)\right]\right) \delta_{U} \delta_{F} \\
& +w_{1}\left(1-\delta_{U}\right) \delta_{F}-L_{F}\left(1-\delta_{F}\right)-\kappa_{F}-c\left(\mu_{F}\right) .
\end{aligned}
$$

With probability $\delta_{U} \delta_{F}$, the outcome of the tournament is not annulled, because no player gets defaulted. In this case, each player receives $w_{2}$ for sure - either directly as loser prize or as part of $w_{1}$ in case of winning and the additional prize spread $\Delta w$ with probability $F\left(d_{U} \mu_{U}-d_{F} \tau \mu_{F}\right)$ or $1-F\left(d_{U} \mu_{U}-d_{F} \tau \mu_{F}\right)$, respectively. With probability $\left(1-\delta_{U}\right) \delta_{F}$ only the underdog gets defaulted so that the favorite is declared the winner of the tournament. With probability $\delta_{U}\left(1-\delta_{F}\right)$ the opposite happens. Each player has direct $\left(\kappa_{i}\right)$ and expected indirect $\operatorname{costs}\left(L_{i}\left(1-\delta_{i}\right)\right)$ of doping $(i=U, F)$. In any case, both players $U$ and $F$ have to bear their investment costs from legal investment, $c\left(\mu_{U}\right)$ and $c\left(\mu_{F}\right)$. Note that doping itself creates a natural trade-off for each player: On the one hand, taking drugs enhances a player's performance and, therefore, also his winning probability. On the other hand, doping implies a positive probability of getting defaulted. 
The first-order conditions for $\mu_{U}^{*}$ and $\mu_{F}^{*}$ show that, if an equilibrium in pure strategies exists at the tournament stage, ${ }^{13}$ it will be asymmetric ${ }^{14}$ and described by the system of equations

$$
\begin{aligned}
& c^{\prime}\left(\mu_{U}^{*}\right)=d_{U} \Delta w f\left(d_{U} \mu_{U}^{*}-d_{F} \tau \mu_{F}^{*}\right) \delta_{U} \delta_{F} \\
& c^{\prime}\left(\mu_{F}^{*}\right)=d_{F} \tau \Delta w f\left(d_{U} \mu_{U}^{*}-d_{F} \tau \mu_{F}^{*}\right) \delta_{U} \delta_{F} .
\end{aligned}
$$

Since marginal costs are increasing due to the convexity of the cost function, each player's equilibrium investment monotonically increases in the prize spread, $\Delta w$, and the probability that the outcome of the tournament is not annulled, $\delta_{U} \delta_{F}$. Eqs. (7) and (8) also show that, given the same level of doping (i.e. $d_{U}=d_{F}$ ), the favorite will always choose higher investment in equilibrium because of a higher marginal probability of winning (i.e. $d_{F} \tau f\left(d_{U} \mu_{U}^{*}-d_{F} \tau \mu_{F}^{*}\right) \delta_{U} \delta_{F}>d_{U} f\left(d_{U} \mu_{U}^{*}-d_{F} \tau \mu_{F}^{*}\right) \delta_{U} \delta_{F}$ due to $\left.\tau>1\right)$.

Note that the impact of doping on investment is twofold: On the one hand, doping increases the productivity of one's investment since $d_{i}$ and $\mu_{i}$ $(i=U, F)$ are complements in the performance function (1). This positive productivity effect enhances investment incentives. On the other hand, doping also has a competition effect: Investment incentives crucially depend on a player's marginal probability of winning and, therefore, on $f\left(d_{U} \mu_{U}^{*}-d_{F} \tau \mu_{F}^{*}\right)$. This term has its maximum at zero because of $f(\cdot)$ 's unique mode. The larger the difference $\left|d_{U} \mu_{U}^{*}-d_{F} \tau \mu_{F}^{*}\right|$, the more uneven will be the competition and the smaller will be the marginal winning probability since the

\footnotetext{
${ }^{13}$ It is well-known in the tournament literature that the existence of pure-strategy equilibria cannot be guaranteed in general; see, e.g., Lazear and Rosen (1981), p. 845, fn. 2; Nalebuff and Stiglitz (1983). Hence, we assume existence throughout the paper. For the parametric case of quadratic costs and uniformly distributed noise, conditions (4) guarantee existence of pure-strategy equilibria.

${ }^{14} \mathrm{On}$ asymmetric equilibria in contest games with heterogeneous players see also Baik (1994) and Nti (1999). However, both authors analyze a logit-form contest and not a rank-order tournament (or probit model).
} 
density monotonically decreases to the tails. Hence, doping makes the competition between the two players more or less uneven. The more uneven the competition (i.e. the larger $\left|d_{U} \mu_{U}^{*}-d_{F} \tau \mu_{F}^{*}\right|$ ), the lower will be the players' investment incentives. Altogether, the overall impact of doping on investments depends on the interplay of the productivity and the competition effect. $^{15}$

Let, for brevity, $\bar{f}:=f\left(d_{U} \mu_{U}^{*}-d_{F} \tau \mu_{F}^{*}\right)$. Then comparative statics lead to the following results: ${ }^{16}$

Proposition 1 Implicit differentiation of the system of Eqs. (7) and (8) yields

$$
\begin{aligned}
& \frac{\partial \mu_{U}^{*}}{\partial d_{U}} \gtrless 0 \text { if } d_{F}^{2} \tau^{2} \bar{f}^{\prime} \bar{f}+\frac{\left[\bar{f}+d_{U} \mu_{U}^{*} \bar{f}^{\prime}\right] c^{\prime \prime}\left(\mu_{F}^{*}\right)}{\Delta w \delta_{U} \delta_{F}} \gtrless 0 \\
& \frac{\partial \mu_{F}^{*}}{\partial d_{F}} \gtrless 0 \text { if } \frac{\left[\bar{f}-d_{F} \mu_{F}^{*} \tau \bar{f}^{\prime}\right] c^{\prime \prime}\left(\mu_{U}^{*}\right)}{\Delta w \delta_{U} \delta_{F}}-d_{U}^{2} \bar{f}^{\prime} \bar{f} \gtrless 0 \\
& \frac{\partial \mu_{U}^{*}}{\partial d_{F}} \gtrless 0 \text { if } d_{U} \mu_{U}^{*} \gtrless d_{F} \tau \mu_{F}^{*} \\
& \frac{\partial \mu_{F}^{*}}{\partial d_{U}} \gtrless 0 \text { if } d_{U} \mu_{U}^{*} \lessgtr d_{F} \tau \mu_{F}^{*} .
\end{aligned}
$$

\section{Proof. See Appendix.}

The results of Proposition 1 show how doping influences the players' equilibrium investments. Obviously, the impact of doping crucially depends on whether $d_{U} \mu_{U}^{*}>d_{F} \tau \mu_{F}^{*}$ or $d_{U} \mu_{U}^{*}<d_{F} \tau \mu_{F}^{*}$, i.e. whether competition takes place at the right-hand side (RHS) or at the left-hand side (LHS) of the convolution $f(\cdot)$. If we are at the LHS, we will have a situation in which the favorite currently has a higher probability of winning than the underdog.

\footnotetext{
${ }^{15}$ If we had an additive production technology so that doping and investment were substitutes in (1), the productivity effect would be absent.

${ }^{16}$ Of course, $d_{U}$ and $d_{F}$ are discrete variables. However, for simplicity here they are treated as if they were continuous.
} 
Let, for the moment, $d$ be smaller than $\tau$ so that talent dominates doping and the competition is quite uneven. As we know from (7) and (8), in this situation we are always at the LHS of $f(\cdot)$ - irrespective of the players' doping decisions.

If now the favorite increases his doping activities he will unambiguously discourage the underdog since the uneven competition becomes even more uneven resulting into $\partial \mu_{U}^{*} / \partial d_{F}<0$. Interestingly, the effect of increased doping by the favorite on his own equilibrium investment is not clear at all. On the one hand, a change from $d_{F}=1$ to $d_{F}=d$ implies that the favorite's investment becomes more productive which leads to increased incentives (productivity effect). On the other hand, incentives significantly decrease due to the competition effect (i.e. the uneven competition is now more uneven which results into lower incentives). As the second comparative-static result of Proposition 1 shows, at the LHS the favorite's investment will decrease as a direct consequence of being doped if the competition effect dominates the productivity effect. If, at the LHS, the underdog's doping activity increases, the underdog will be back into the race. Now competition becomes more even, which leads to higher equilibrium investments by both players according to the last result of Proposition 1. Whereas the favorite's investment solely increases due to the competition effect, both the productivity and the competition effect make the underdog choose a higher investment level.

Now consider possible comparative statics at the RHS of $f(\cdot)$. As we know from conditions (7) and (8), the RHS can only become relevant if $d_{U}=d>\tau$ and $d_{F}=1$, i.e. if the underdog is doped and the favorite not, and doping dominates talent. Proposition 1 indicates that for the RHS we find results similar to the findings for the LHS. However, it is important to emphasize that these considerations are only true for rather small changes 
of $d_{F}$ since the comparative statics exclusively hold for a marginal change of $d_{F}$. Note that starting at the RHS and increasing $d_{F}$ from 1 to $d$ would lead to the LHS of the distribution. Of course, in the new situation the favorite's investments are larger than the underdog's investments, and the favorite now has a higher winning probability than the underdog, contrary to the starting point.

\subsection{The Doping Stage}

At the first stage of the game, both players simultaneously have to decide on their doping activities while knowing that they will choose $\mu_{U}^{*}\left(d_{U}, d_{F}\right)$ and $\mu_{F}^{*}\left(d_{U}, d_{F}\right)$ according to $(7)$ and (8) in the subsequent tournament stage. There are four possible equilibria - a mutual doping equilibrium, an equilibrium in which no player is doped, an equilibrium in which only the underdog is doped, and an equilibrium in which only the favorite is doped. A complete analysis of all four equilibria would be beyond the scope of this paper. We therefore focus on the most interesting case of a mutual-doping equilibrium $\left(d_{U}^{*}, d_{F}^{*}\right)=(d, d)$ and discuss the players' incentives to unilaterally deviate to no-doping.

The strategies $\left(d_{U}^{*}, d_{F}^{*}\right)=(d, d)$ will form an equilibrium, if the underdog (favorite) does not want to deviate to $d_{U}=1\left(d_{F}=1\right)$ and a corresponding optimal $\mu_{U}\left(\mu_{F}\right)$ given $d_{F}=d\left(d_{U}=d\right)$ and $\mu_{F}^{*}(d, d)\left(\mu_{U}^{*}(d, d)\right)$. Formally, 
$\left(d_{U}^{*}, d_{F}^{*}\right)=(d, d)$ will be an equilibrium if and only if

$$
\begin{aligned}
& E U_{U}\left(\mu_{U}^{*}(d, d) ; \mu_{F}^{*}(d, d), d, d\right) \geq E U_{U}\left(\hat{\mu}_{U} ; \mu_{F}^{*}(d, d), 1, d\right) \text { with } \\
& \hat{\mu}_{U}=\underset{\mu_{U}}{\arg \max } E U_{U}\left(\mu_{U} ; \mu_{F}^{*}(d, d), 1, d\right) \quad \text { and } \\
& E U_{F}\left(\mu_{F}^{*}(d, d) ; \mu_{U}^{*}(d, d), d, d\right) \geq E U_{F}\left(\hat{\mu}_{F} ; \mu_{U}^{*}(d, d), d, 1\right) \text { with } \\
& \hat{\mu}_{F}=\underset{\mu_{F}}{\arg \max } E U_{F}\left(\mu_{F} ; \mu_{U}^{*}(d, d), d, 1\right) .
\end{aligned}
$$

Using objective functions (5) and (6) yields the following proposition:

Proposition 2 Let $\hat{\mu}_{U}$ and $\hat{\mu}_{F}$ be defined by (9) and (10). Then there will be a doping equilibrium $\left(d_{U}^{*}, d_{F}^{*}\right)=(d, d)$ if and only if

$$
\begin{gathered}
\delta \Delta w\left[F\left(d \mu_{U}^{*}(d, d)-d \tau \mu_{F}^{*}(d, d)\right) \delta-F\left(\hat{\mu}_{U}-d \tau \mu_{F}^{*}(d, d)\right)\right] \\
\geq(1-\delta)\left(w_{1}-\delta \Delta w\right)+L_{U}(1-\delta)+\bar{\kappa}_{U}+\left[c\left(\mu_{U}^{*}(d, d)\right)-c\left(\hat{\mu}_{U}\right)\right] \quad \text { and } \\
\\
\quad \delta \Delta w\left[F\left(d \mu_{U}^{*}(d, d)-\tau \hat{\mu}_{F}\right)-F\left(d \mu_{U}^{*}(d, d)-d \tau \mu_{F}^{*}(d, d)\right) \delta\right] \\
\geq(1-\delta) w_{1}+L_{F}(1-\delta)+\bar{\kappa}_{F}+\left[c\left(\mu_{F}^{*}(d, d)\right)-c\left(\hat{\mu}_{F}\right)\right] .
\end{gathered}
$$

While condition (11) guarantees that the underdog does not deviate from the doping equilibrium, condition (12) ensures that the favorite also prefers to be doped given a doped opponent. If we neglect the indirect and direct costs from doping for a moment - of course, if $L_{i}(1-\delta)+\bar{\kappa}_{i}(i=U, F)$ becomes sufficiently large, neither player will choose doping -, there are three effects which determine the doping decision of the two players: The first effect can be referred to as likelihood effect being characterized by the expression in brackets at the left-hand side of (11) and (12), respectively. This expression describes the change of a player's winning probability when choosing doping 
instead of no-doping given that the opponent is doped. Consider, for example, condition (11) for the underdog. Note that here we have $\hat{\mu}_{U}<\mu_{U}^{*}(d, d)$ : In a doping equilibrium $\left(d_{U}^{*}, d_{F}^{*}\right)=(d, d)$, we are at the LHS of the probability distribution with $d_{U} \mu_{U}^{*}<d_{F} \tau \mu_{F}^{*}$. For given $d_{F}$ and $\mu_{F}^{*}$, deviating from $d_{U}=d$ to a slightly lower doping level leads to a change in investment which can be calculated by implicit differentiation of (A1) (see the proof of Proposition 1 in the Appendix):

$$
\frac{\partial \mu_{U}}{\partial d_{U}}=-\frac{\Delta w \delta_{U} \delta_{F}}{E U_{U}^{\prime \prime}}\left(\bar{f}+d_{U} \mu_{U} \bar{f}^{\prime}\right)>0 \quad \text { at LHS }
$$

with $\bar{f}:=f\left(d_{U} \mu_{U}-d_{F} \tau \mu_{F}\right)$. Hence, a marginal decrease of $d_{U}$ would lead to a lower investment level so that the difference $\left|d_{U} \mu_{U}-d_{F} \tau \mu_{F}\right|$ becomes even larger. Decreasing $d_{U}=d$ to $d_{U}=1$ therefore unambiguously implies $\hat{\mu}_{U}<\mu_{U}^{*}(d, d) .{ }^{17}$ Since the cdf $F(\cdot)$ is monotonically increasing, for $\delta$ not being too small, ${ }^{18}$ the expression in brackets at the left-hand side of (11) is positive so that the likelihood effect supports a doping equilibrium: doping becomes attractive in order to increase one's likelihood of winning.

The second effect can be called cost effect. It is characterized by the expression in brackets at the right-hand side of (11) and (12), respectively. This expression measures the change of a player's investment costs when choosing doping instead of no-doping given that the opponent is doped. The equilibrium conditions point out that the likelihood effect and the cost effect work into opposite directions: If doping leads to higher investments and higher probabilities of winning, the players' investment costs will rise so that the cost effect makes mutual doping less attractive.

\footnotetext{
${ }^{17}$ The intuition for this result comes from the productivity effect and the competition effect mentioned in Subsection 3.1 which both work into the same direction.

${ }^{18}$ According to Haugen (2004), the probability of being caught in case of doping is rather low in practice.
} 
Third, we have the term $(1-\delta)\left(w_{1}-\delta \Delta w\right)$ at the right-hand side of (11) and the term $(1-\delta) w_{1}$ at the right-hand side of (12). These terms correspond to an effect which can be characterized as windfall-profit effect. Note that each player will strictly gain if his opponent - contrary to himself gets defaulted because of detected doping. In this situation, the opponent has no positive income whereas the other player receives the high winner prize as windfall profit irrespective of his relative performance. If a player chooses doping instead of no-doping given that his opponent is doped, the player's probability of getting defaulted increases from zero to $1-\delta$. As a direct consequence, the player's expected windfall profit decreases by multiplication with $(1-\delta)$. Hence, the windfall-profit effect always works against a doping equilibrium.

To sum up, whether a doping equilibrium will emerge or not, crucially depends on the interplay of the three effects, the magnitude of direct and indirect doping costs, the reliability of the doping test and the magnitude of the tournament prizes. If the doping test is highly reliable so that the non-detection probability $\delta$ tends to zero, both the likelihood effect and the cost effect will diminish - the cost effect since all investments tend to zero, ${ }^{19}$ and the likelihood effect since a possibly higher winning probability is totally offset by the fact that getting defaulted is almost sure. Furthermore, the importance of each effect depends on the specific sport under consideration and on the concrete meaning of the diverse variables. In particular, if $\mu_{U}$ and $\mu_{F}$ only capture the actual effort exerted in a sport contest so that $c(\cdot)$ describes the disutility of effort, the cost effect can be neglected in most sports. In this case, each athlete will typically do his best without thinking about the corresponding exertions. If, however, $\mu_{U}$ and $\mu_{F}$ describe time

\footnotetext{
${ }^{19}$ This can be seen from the first-order conditions (7) and (8), and the respective firstorder conditions for $\hat{\mu}_{U}$ and $\hat{\mu}_{F}$.
} 
consuming training, the cost effect may become very important.

Finally, we can analyze the influence of the tournament prizes $w_{1}$ and $w_{2}$ on (11) and (12). At first sight, one might expect that high winner prizes provoke more doping and are therefore detrimental. However, the equilibrium conditions show that both the likelihood effect and the windfall-profit effect directly increase in $w_{1}$ so that the influence of the winner prize is not clear at all. ${ }^{20}$ The left-hand sides of (11) and (12) point out that the prize spread $\Delta w$ is influential. From standard tournament results we know that investment incentives do not depend on the absolute values of $w_{1}$ and $w_{2}$ but on the spread $\Delta w$ (see also Eqs. (7) and (8)). Conditions (11) and (12) show that, given a positive likelihood effect (i.e. the left-hand sides of (11) and (12) are positive), the attractiveness of doping also increases in the prize spread. Hence, a tournament organizer can try to prevent doping by setting only a moderate prize spread. ${ }^{21}$ Note that this decision would also lessen the perils of other forms of cheating like sabotage (see Lazear 1989), but comes at the cost that productive incentives would also decrease. Since $\Delta w=w_{1}-w_{2}$, mutual doping can be prevented by a high loser prize $w_{2}$. This policy would have two effects: First, the prize spread and hence the expected gains from doping would decrease. Second, by inspection of (5) and (6) we can see that each player earns an expected base salary $w_{2} \delta_{U} \delta_{F}$. If $w_{2}$ is high, the players might prefer not to take drugs in order to receive the base salary $w_{2}$ with higher probability.

In order to derive explicit solutions and to obtain further insights on the influence of the parameters on the doping equilibrium $\left(d_{U}^{*}, d_{F}^{*}\right)=(d, d)$, we now use the uniform distribution for the noise variables and the quadratic

\footnotetext{
${ }^{20}$ Note that the prizes also have an indirect influence on the likelihood and the cost effect since equilibrium investments depend on the prize spread $\Delta w$.

${ }^{21}$ See also Eber and Thépot (1999), 441-442. However, up to now, it is not clear whether a tournament organizer really wants to prevent doping.
} 
cost function described in Section 2. Computing the respective equilibrium conditions leads to the following result:

Corollary 1 Let $c\left(d^{2} \delta-\tau^{2}\right)>d^{2} \Delta w \tau^{2} \gamma^{2} \delta^{2}\left(\tau^{2}-1\right)$, costs be quadratic and noise be uniformly distributed. A doping equilibrium will exist, if and only if

$$
\begin{gathered}
(1-\delta) \delta \Delta w+\frac{c \Delta w \delta\left(c-\gamma^{2} \Delta w d^{2} \delta^{2}\right)}{2\left(\gamma^{2} \Delta w d^{2} \delta^{2}\left(\tau^{2}-1\right)+c\right)^{2}}\left(\delta-\frac{\left(c-\gamma^{2} \Delta w \delta^{2} d^{2}\right)}{\left(c-\gamma^{2} \Delta w \delta\right)}\right) \\
>(1-\delta) w_{1}+L_{U}(1-\delta)+\bar{\kappa}_{U} \quad \text { and } \\
\delta \Delta w-\frac{c \Delta w \delta\left(\delta\left(\gamma^{2} \Delta w d^{2} \delta^{2} \tau^{2}+c\right)+\frac{\left(d^{2} \Delta w \gamma^{2} \delta^{2}\left(\tau^{2}-2\right)+c\right)^{2}}{\left(c-\Delta w \tau^{2} \gamma^{2} \delta\right)}\right)}{2\left(\gamma^{2} \Delta w d^{2} \delta^{2}\left(\tau^{2}-1\right)+c\right)^{2}} \\
>(1-\delta) w_{1}+L_{F}(1-\delta)+\bar{\kappa}_{F} .
\end{gathered}
$$

\section{Proof. See Appendix.}

Condition (13) describes the underdog's equilibrium condition, whereas inequality (14) ensures that the favorite does not want to unilaterally deviate from mutual doping. As the proof of the corollary shows, deviating from $d_{i}=d$ to $d_{i}=1(i=U, F)$ has similar consequences for both players. On the one hand, their efforts and, therefore effort costs decrease, on the other hand the same is true for their winning probabilities. Hence, we find a clear trade-off between cost effect and likelihood effect as has been indicated in the discussion of Proposition 2.

The general parameter condition given at the beginning of Corollary 1 as well as condition (13) show that there exists a cut-off $\hat{\tau}$ so that $(d, d)$ will never be an equilibrium if $\tau>\hat{\tau}$. Thus, for sufficiently high degrees of heterogeneity mutual doping will never be stable. The intuition for this result is the following: Recall that winning the tournament depends on a player's 
talent, his investment and his doping decision. If the players' talents are very different, a player can hardly influence the outcome of the tournament by his doping decision. However, doping is still dangerous for a player since it can lead to disqualification. Hence, players rationally decide not to be doped.

The equilibrium conditions point out that if doping tests are highly reliable (i.e. if the non-detection probability $\delta$ tends to zero), mutual doping will never be an equilibrium. In this situation, the perils of getting defaulted are so high that the choice of $d$ cannot be attractive at all.

Conditions (13) and (14) also show the influence of indirect and direct doping costs, $L_{i}(1-\delta)+\bar{\kappa}_{i}(i=U, F)$. Intuitively, the direct costs of doping, $\bar{\kappa}_{i}$, should not be too different for both players as expenditures for drugs and costs due to affected health typically do not depend on whether a player is an underdog or a favorite. However, the expected indirect costs, $L_{i}(1-\delta)$, should crucially depend on the type of player. If these indirect costs mainly characterize the expected loss of reputation, $L_{U}$ should be very different from $L_{F}$. Typically, the loss of advertising revenues will be very large if a clear favorite (a "superstar") is detected when being doped whereas advertising revenues of underdogs are rather small. Moreover, superstars often get money for participating in a contest. Of course, this future income will also get lost if getting defaulted. Altogether, if $L_{F}$ is quite large relative to $L_{U}$ doping will be less attractive for a favorite as he has too much at the stake.

Finally, we can investigate whether the underdog or the favorite is more likely to deviate from mutual doping. When subtracting the left-hand side of (14) from the left-hand-side of (13) and ignoring for the moment the influence of doping costs we obtain:

Corollary 2 Let $L_{U}(1-\delta)+\bar{\kappa}_{U}=L_{F}(1-\delta)+\bar{\kappa}_{F}$, costs be quadratic and noise be uniformly distributed. The underdog will be more likely to deviate 
from mutual doping than the favorite if

$$
\frac{\Delta w^{2} \gamma^{2} \delta^{2}\left(\tau^{2}-1\right)\left(c^{3} X+c^{2} Y+c Z-2 d^{4} \Delta w^{3} \tau^{2} \gamma^{6} \delta^{6}\left(\tau^{2}-1\right)\right)}{2\left(c-\Delta w \gamma^{2} \delta\right)\left(c-\Delta w \tau^{2} \gamma^{2} \delta\right)\left(d^{2} \Delta w \gamma^{2} \delta^{2}\left(\tau^{2}-1\right)+c\right)^{2}}<0
$$

with

$$
\begin{aligned}
X & :=-(3 \delta-2) d^{2} \delta+1 \\
Y \quad: & =d^{2} \Delta w \gamma^{2} \delta^{2}\left(3 \delta+(2 \delta-3) d^{2} \delta+\left(3+d^{2}(1-2 \delta)\right) \delta \tau^{2}-4\right) \\
Z & :=-d^{2} \Delta w^{2} \gamma^{4} \delta^{4}\left(d^{2}\left(\tau^{2}+2 \delta-4-2 \tau^{4} \delta\right)+3 \tau^{2}\right) .
\end{aligned}
$$

The corollary points out that if the cost parameter is rather large (which indeed should be the case to guarantee concave objective functions; see (4)) only the impact of doping $d$ and the magnitude of the non-detection probability $\delta$ determine which type of player is more likely to deviate from the possible doping equilibrium: In this case, the sign of the coefficient $X$ for the cubic term of the cost parameter is decisive, and $X$ only depends on $d$ and $\delta$. In particular, if $\delta$ is sufficiently large, the cubic term $c^{3} X$ will be negative; if $\delta=1$ it will be negative for all feasible values of $d$ because of (4). However, if $\delta$ is sufficiently small, $c^{3} X$ will be positive. Thus, if the doping test is rather unreliable (very reliable), the underdog (the favorite) will more likely deviate from mutual doping than the favorite (underdog).

Since a doping equilibrium will not exist, if doping tests are highly reliable (see Corollary 1), we should expect that, in practice, an underdog less often prefers mutual doping than a favorite. ${ }^{22}$ The intuition for this result

\footnotetext{
${ }^{22}$ In the model by Berentsen (2002) for certain parameter constellations there exists a mixed-strategy equilibrium where the favorite will be doped with a higher probability than the underdog if doping is sufficiently effective, the costs of doping are sufficiently small and the winner prize is sufficiently large.
} 
comes from the interplay of the cost effect and the likelihood effect: ${ }^{23}$ The proof of Corollary 1 has shown that deviating from $d_{F}=d$ to $d_{F}=1$ strictly harms the favorite as it significantly decreases $F$ 's probability of winning; after the deviation we are at the RHS of the distribution so that $F$ now is less likely to win the contest compared to $U$ whereas he was more likely to win before. In addition, competition might become closer between the players which then implies that $F$ cannot gain very much from reduced investment costs. The situation is different for the underdog. Deviating from $d_{U}=d$ to $d_{U}=1$ would also decrease his probability of winning, but now the uneven competition unambiguously becomes even more uneven. This effect may lead to significantly reduced investment costs which can outweigh part of the disadvantage from decreased winning probability. In other words, doping can be profitable for a favorite for two reasons - increasing the probability of winning and reducing investment costs by making competition more uneven. Hence, both effects would work into the same direction. However, for the underdog choosing doping often leads to countervailing effects: While increasing the likelihood of winning, coming back into the race might lead to increased competition and, hence, increased investment costs.

\section{Discussion}

\section{Ex-ante versus ex-post testing}

As we have seen in Section 3, the organizer of a tournament can decrease the players' incentives to take drugs by choosing a high loser prize or a low prize spread. However, up to now it is not clear whether the organizer would really be interested in preventing the consumption of drugs. On the one

\footnotetext{
${ }^{23}$ Note that Corollary 2 only focuses on these two effects.
} 
hand, doping and the disqualification of players who have consumed drugs harms the reputation of a specific sport which may imply fewer spectators or lower revenues from the selling of broadcasting rights. On the other hand, the organizer may be interested in high performances $q_{i}$ and high investments $\mu_{i}^{*}$. For the moment, let us assume that the organizer is interested in preventing the consumption of drugs and that he can choose between ex-post testing after the tournament (as in Section 3) and ex-ante testing before the tournament starts. In the case of ex-ante testing, the doping test takes place between the two stages of the game which have been discussed in the previous section (i.e., after the doping decision but before the players choose investment). ${ }^{24}$ In the following, we will discuss the question, whether the organizer of the tournament should prefer ex-ante or ex-post training in order to combat doping.

In the case of ex-ante testing, the objective function of the underdog at the tournament stage is given by

$$
\begin{aligned}
E U_{U}\left(\mu_{U} ; \mu_{F}, d_{U}, d_{F}\right)= & \left(w_{2}+\Delta w F\left(d_{U} \mu_{U}-d_{F} \tau \mu_{F}\right)-c\left(\mu_{U}\right)\right) \delta_{U} \delta_{F} \\
& +w_{1} \delta_{U}\left(1-\delta_{F}\right)-L_{U}\left(1-\delta_{U}\right)-\kappa_{U}
\end{aligned}
$$

and that of the favorite by

$$
\begin{aligned}
E U_{F}\left(\mu_{F} ; \mu_{U}, d_{U}, d_{F}\right)= & \left(w_{2}+\Delta w\left[1-F\left(d_{U} \mu_{U}-d_{F} \tau \mu_{F}\right)\right]-c\left(\mu_{F}\right)\right) \delta_{U} \delta_{F} \\
& +w_{1}\left(1-\delta_{U}\right) \delta_{F}-L_{F}\left(1-\delta_{F}\right)-\kappa_{F} .
\end{aligned}
$$

The important difference of (15) and (16) compared to the objective functions (5) and (6) is given by the fact that if using ex-ante testing the tournament

\footnotetext{
${ }^{24}$ In practice, if $\mu_{U}$ and $\mu_{F}$ denote efforts ex-ante testing will mean a test directly before the tournament. If the investments stand for (final) training, ex-ante testing will take place in an early training period.
} 
will not take place in any case. The two players will only compete (by choosing investments) with probability $\delta_{U} \delta_{F}$. With probability $1-\delta_{U} \delta_{F}$ there will be no tournament, no investments and no investment costs because of the disqualification of at least one contestant. ${ }^{25}$

In analogy to Section 3, the first-order conditions yield

$$
\begin{aligned}
& c^{\prime}\left(\mu_{U}\right)=d_{U} \Delta w f\left(d_{U} \mu_{U}-d_{F} \tau \mu_{F}\right) \\
& c^{\prime}\left(\mu_{F}\right)=d_{F} \tau \Delta w f\left(d_{U} \mu_{U}-d_{F} \tau \mu_{F}\right) .
\end{aligned}
$$

By comparing these conditions with (7) and (8) we can see that, for a given pair $\left(d_{U}, d_{F}\right)$, equilibrium investments will always be larger under ex-ante than under ex-post testing. The intuition for this result is straightforward. In the case of ex-post testing, players do not know whether the tournament will be annulled afterwards so that their investments are lost. This leads to lower incentives which decrease in $\left(1-\delta_{U}\right)$ and $\left(1-\delta_{F}\right)$. Altogether, we have the following trade-off: Under ex-post testing, there is always a tournament, but investments are lower than under ex-ante testing. However, under exante testing, investments are larger given that a tournament takes place, but this event is risky.

\section{Endogenous tournament prizes}

Until now, tournament prizes have been assumed to be exogenous in order to focus on the doping game between the two heterogeneous players. Discussing endogenous prizes seems to be problematic in this context. First, we have to specify the objective function of the organizer of the tourna-

\footnotetext{
${ }^{25}$ Note that if one player gets defaulted under ex-ante testing, the other player will receive the winner prize although no tournament takes place. This special situation is due to the fact that we consider a two-person tournament. However, for $n>2$ contestants we would have a similar effect. In that case, the remaining contestants' winning probability would increase by the disqualification of a player.
} 
ment. In the economic literature on sport contests, several possible objective functions have been discussed. ${ }^{26}$ The organizer may be interested in competitive balance to guarantee an attractive competition and, therefore, high revenues from the selling of broadcasting rights. Alternatively, the organizer may want to maximize total expected performance minus tournament prizes, $E\left[q_{U}+q_{F}\right]-w_{1}-w_{2}$. As another alternative, the organizer's revenues may increase in the realization of top performances (e.g., beating records) so that the organizer may want to maximize $\max \left\{q_{U}, q_{F}\right\}-w_{1}-w_{2}$. To sum up, it is not quite clear how the correct objective function should look like.

Second, given a certain objective function, it is not obvious whether the organizer wants to prevent doping or not. For example, the organizer may want to implement doping by the underdog but prevent doping by the favorite in order to increase competitive balance. As we know from the discussion above, increased competitive balance enhances overall performance of both players (see the competition effect mentioned in Subsection 3.1) and may, in turn, lead to a maximum individual performance. In this scenario, unilateral doping by the underdog would be optimal for the organizer under either objective function. However, if the reputation of a certain sport is fundamentally harmed by detected doping, preventing doping may be the organizer's primary aim in any case.

When calculating optimal prizes, the organizer would choose $w_{1}$ and $w_{2}$ in order to maximize his objective function subject to the players' incentive constraints (7) and (8), the two participation constraints $E U_{U}\left(\mu_{U}^{*} ; \mu_{F}^{*}, d_{U}^{*}, d_{F}^{*}\right) \geq$ $\bar{u}_{U}$ and $E U_{F}\left(\mu_{F}^{*} ; \mu_{U}^{*}, d_{U}^{*}, d_{F}^{*}\right) \geq \bar{u}_{F}$ with $\bar{u}_{i}$ denoting players $i$ 's $(i=U, F)$ reservation utility, and two constraints implementing a favored pair $\left(d_{U}^{*}, d_{F}^{*}\right)$. If, for example, the organizer wants to implement a doping equilibrium $(d, d)$

\footnotetext{
${ }^{26}$ See, for example, Fort and Quirk (1995), Szymanski (2003), Szymanski and Kesenne (2004), Falconieri, Palomino and Sákovics (2004), Kesenne (2005).
} 
and the players are not wealth-restricted (i.e., there is no limited liability), the organizer will choose the lowest possible loser prize $w_{2}^{*}$ which makes the participation constraint of the player with the lower expected utility just bind. This loser prize both guarantees that the organizer's labor costs become as low as possible and supports the doping conditions (11) and (12). Furthermore, a rather large prize spread $\Delta w^{*}$ is chosen by the organizer in order to support conditions (11) and (12), and to implement considerable investment levels according to (7) and (8). Note that despite risk neutral players and unlimited liability, the organizer does not want to implement first-best effort because one player earns a positive rent due to the players' heterogeneity.

\section{Welfare analysis}

For a welfare analysis, similar problems arise as in the discussion of optimal tournament prizes since first we have to define welfare in the given context. Following the analysis of Konrad (2005) we can, for example, define welfare as the difference of expected total output minus total costs. Hence, in our model welfare would be

$$
W=\sum_{i \in\{U, F\}}\left(d_{i} t_{i} \mu_{i}+E\left[\varepsilon_{i}\right]-c\left(\mu_{i}\right)-L_{i}\left(1-\delta_{i}\right)-\kappa_{i}\right)
$$

Konrad (2005) also considers a model in which the doping and the investment inputs are complements in the production function. Furthermore, both inputs have separable convex cost functions, but doping does not imply the possibility to get defaulted and players are homogeneous. There is only one prize in the Konrad model which is given to the tournament winner. Konrad shows that if the tournament prize that maximizes welfare is chosen, mutual doping will be welfare improving. In our model with heterogeneous players, 
we find the same curious result:

Proposition 3 Let $L_{i}\left(1-\delta_{i}\right)+\kappa_{i} \rightarrow 0(i=U, F)$. If tournament prizes are chosen which maximize welfare $W$, mutual doping $\left(d_{U}, d_{F}\right)=(d, d)$ will be welfare enhancing.

Proof. The first-best investments for both players, $\mu_{i}^{F B}(i=U, F)$, which maximize $W$ as given in (17), are

$$
c^{\prime}\left(\mu_{i}^{F B}\right)=d_{i} t_{i}
$$

We know that players choose investments according to (7) and (8). Comparing (18) with (7) and (8) shows that the prize spread

$$
\Delta w^{F B}=\frac{1}{f\left(d_{U} \mu_{U}^{*}-d_{F} \tau \mu_{F}^{*}\right) \delta_{U} \delta_{F}}
$$

implements first-best incentives for both players for any pair $\left(d_{U}, d_{F}\right)$. Hence, the only influence of doping on welfare remains via $d_{i}(i=U, F)$ in (17) so that $d_{U}=d_{F}=d$ leads to maximum welfare.

If welfare maximizing tournament prizes are chosen, these prizes will always be adjusted to the doping levels $\left(d_{U}, d_{F}\right)$. In other words, incentives are not influenced by doping under optimal prizes. In this case, doping $d$ will only increase both players' aggregate performance and therefore overall welfare. Of course, this curious result crucially depends on the definition of welfare and the assumption that direct and indirect costs of doping are negligible $\left(L_{i}\left(1-\delta_{i}\right)+\kappa_{i} \rightarrow 0\right)$. If overall welfare is reduced by detected consumption of drugs, since the spectators' utilities decrease, and/or by the players' costs of doping (loss of reputation, expenditures for drugs, affected health) mutual doping will not necessarily be welfare maximizing. In particular, we might 
have a rat-race like situation in which both players become stronger because of doping but the relative strength of the players does not really change so that competitive balance remains the same while overall costs of doping rise significantly.

\section{Conclusion}

Although doping in contests is an important topic from an economic perspective, it is not clear whether the utilization of illegal resources should be generally prevented from the viewpoint of the contest organizer or from society's perspective. Hence, in this paper we focus on a stylized doping game between two players in order to analyze the determinants of doping and possible alternatives for preventing doping given that such behavior is welfare reducing.

Contrary to most of the existing doping models, we assume heterogeneous players who choose both doping and a legal input (e.g., effort or training) and face a positive probability of getting defaulted in case of doping. Furthermore, we assume that doping, the legal input and ability are complements in the players' production functions. We identify three effects which determine the attractiveness of taking drugs: a likelihood effect (i.e. doping increases one's own probability of winning if not getting defaulted), a cost effect (i.e. doping influences the exertion of the legal input and hence costs), a windfallprofit effect (i.e. if one player is disqualified, the other player wins for sure). If the expected costs of getting defaulted are not too high for the favorite, under reasonable assumptions the favorite tends more likely to use drugs than the underdog, and mutual doping by both players may be welfare enhancing.

In a next step, it would be interesting to analyze the players' doping 
decision within a closed model where the organizer of the tournament wants to maximize a certain objective function and doping is a continuous variable. However, this step will not be a trivial one since there exist several trade-offs when discussing doping in such a context. In particular, more doping will typically lead to a higher probability of getting defaulted and to higher health costs of the players. Hence, it will be difficult to find out the optimal level of doping from both the organizer's and society's perspective. Perhaps, it will be helpful to concentrate on a specific application, for example on doping in a certain professional sport. By this it might be easier to formulate a concrete objective function for the organizer of the contest since in professional sports the selling of broadcasting rights is of major importance. 


\section{Appendix:}

Proof of Proposition 1:

The two first-order conditions (7) and (8) can be written as

$$
\begin{aligned}
& F_{1}:=d_{U} \Delta w f\left(d_{U} \mu_{U}^{*}-d_{F} \tau \mu_{F}^{*}\right) \delta_{U} \delta_{F}-c^{\prime}\left(\mu_{U}^{*}\right)=0 \\
& F_{2}:=d_{F} \tau \Delta w f\left(d_{U} \mu_{U}^{*}-d_{F} \tau \mu_{F}^{*}\right) \delta_{U} \delta_{F}-c^{\prime}\left(\mu_{F}^{*}\right)=0 .
\end{aligned}
$$

Let

$$
\begin{aligned}
|J| & =\left|\begin{array}{cc}
\frac{\partial F_{1}}{\partial \mu_{U}^{*}} & \frac{\partial F_{1}}{\partial \mu_{F}^{*}} \\
\frac{\partial F_{2}}{\partial \mu_{U}^{*}} & \frac{\partial F_{2}}{\partial \mu_{F}^{*}}
\end{array}\right| \\
& =E U_{U}^{\prime \prime} \cdot E U_{F}^{\prime \prime}+d_{U}^{2} d_{F}^{2} \tau^{2} \Delta w^{2}\left(\bar{f}^{\prime}\right)^{2} \delta_{U}^{2} \delta_{F}^{2}>0
\end{aligned}
$$

be the Jacobian determinant with $E U_{i}^{\prime \prime}$ denoting the second derivative of $E U_{i}$ with respect to $\mu_{i}$ which has to be negative due to the second-order condition. Then, implicit differentiation gives

$$
\begin{aligned}
\frac{\partial \mu_{U}^{*}}{\partial d_{U}} & =\frac{\Delta w^{2} \delta_{U}^{2} \delta_{F}^{2}}{|J|}\left(d_{F}^{2} \tau^{2} \bar{f}^{\prime} \bar{f}+\frac{\left[\bar{f}+d_{U} \mu_{U}^{*} \bar{f}^{\prime}\right] c^{\prime \prime}\left(\mu_{F}^{*}\right)}{\Delta w \delta_{U} \delta_{F}}\right) \\
\frac{\partial \mu_{F}^{*}}{\partial d_{F}} & =\frac{\Delta w^{2} \delta_{U}^{2} \delta_{F}^{2} \tau}{|J|}\left(\frac{\left[\bar{f}-d_{F} \mu_{F}^{*} \tau \bar{f}^{\prime}\right] c^{\prime \prime}\left(\mu_{U}^{*}\right)}{\Delta w \delta_{U} \delta_{F}}-d_{U}^{2} \bar{f}^{\prime} \bar{f}\right) \\
\frac{\partial \mu_{U}^{*}}{\partial d_{F}} & =-\frac{\Delta w^{2} \delta_{U}^{2} \delta_{F}^{2} \tau d_{U} \bar{f}^{\prime}}{|J|}\left(\frac{\mu_{F}^{*} c^{\prime \prime}\left(\mu_{F}^{*}\right)}{\Delta w \delta_{U} \delta_{F}}+d_{F} \tau \bar{f}\right) \\
\frac{\partial \mu_{F}^{*}}{\partial d_{U}} & =\frac{\Delta w^{2} \delta_{U}^{2} \delta_{F}^{2} \tau d_{F} \bar{f}^{\prime}}{|J|}\left(\frac{\mu_{U}^{*} c^{\prime \prime}\left(\mu_{U}^{*}\right)}{\Delta w \delta_{U} \delta_{F}}+d_{U} \bar{f}\right) .
\end{aligned}
$$

Note that $f(\cdot)$ has a unique mode at zero so that $\bar{f}^{\prime} \gtrless 0$ for $d_{U} \mu_{U}^{*} \lessgtr d_{F} \tau \mu_{F}^{*}$.

Proof of Corollary 1:

First, consider the candidate equilibrium $\left(d_{U}, d_{F}\right)=(d, d)$ and $\left(\mu_{U}, \mu_{F}\right)=$ $\left(\mu_{U}(d, d), \mu_{F}(d, d)\right)$. Using the quadratic cost function and the LHS of (2), 
the first-order conditions (7) and (8) yield: ${ }^{27}$

$\mu_{U}(d, d)=\frac{\Delta w \gamma d \delta^{2}}{\gamma^{2} \Delta w d^{2}\left(\tau^{2}-1\right) \delta^{2}+c}$ and $\mu_{F}(d, d)=\frac{\Delta w \gamma d \tau \delta^{2}}{\gamma^{2} \Delta w d^{2}\left(\tau^{2}-1\right) \delta^{2}+c}$

In this situation, player $U$ 's expected utility amounts to ${ }^{28}$

$$
\begin{aligned}
E U_{U}(d, d)= & \delta^{2}\left(w_{2}+\Delta w\left(\gamma\left(d \mu_{U}-d \tau \mu_{F}\right)+\frac{\gamma^{2}}{2}\left(d \mu_{U}-d \tau \mu_{F}\right)^{2}+\frac{1}{2}\right)\right) \\
& +w_{1}(1-\delta) \delta-L_{U}(1-\delta)-\bar{\kappa}_{U}-\frac{c}{2} \mu_{U}^{2} \\
= & w_{1} \delta-\Delta w \delta^{2}+\frac{\delta^{2} \Delta w c\left(c-\gamma^{2} \Delta w d^{2} \delta^{2}\right)}{2\left(\gamma^{2} \Delta w d^{2} \delta^{2}\left(\tau^{2}-1\right)+c\right)^{2}}-L_{U}(1-\delta)-\bar{\kappa}_{U}
\end{aligned}
$$

and that of player $F$ to

$$
E U_{F}(d, d)=w_{1} \delta-\frac{c \Delta w \delta^{2}\left(\gamma^{2} \Delta w d^{2} \delta^{2} \tau^{2}+c\right)}{2\left(\gamma^{2} \Delta w d^{2} \delta^{2}\left(\tau^{2}-1\right)+c\right)^{2}}-L_{F}(1-\delta)-\bar{\kappa}_{F} .
$$

We now have to check whether any of the two players wants to deviate from $\left(d_{U}, d_{F}\right)=(d, d)$. Let us start with the underdog. Given $d_{F}=d$ and $\mu_{F}=\mu_{F}(d, d)$, in case of deviation the underdog would choose $d_{U}=1$ and that value of $\mu_{U}$ that maximizes his expected utility in the given situation. Let $\hat{\mu}_{U}$ denote this investment level. From the discussion of Proposition 2 we know that $\hat{\mu}_{U}<\mu_{U}(d, d)$. Hence, for the case of deviation of $U$, again only the LHS of the probability distribution becomes relevant, and we obtain

$$
\begin{aligned}
\hat{\mu}_{U}= & \underset{\mu_{U}}{\arg \max } \delta \Delta w\left(\gamma\left(\mu_{U}-d \tau \mu_{F}(d, d)\right)+\frac{\gamma^{2}}{2}\left(\mu_{U}-d \tau \mu_{F}(d, d)\right)^{2}+\frac{1}{2}\right) \\
& +\delta w_{2}+w_{1}(1-\delta)-\frac{c}{2} \mu_{U}^{2}
\end{aligned}
$$

${ }^{27}$ Note that $\left(d \mu_{U}(d, d)-d \tau \mu_{F}(d, d)\right) \in\left(-\frac{1}{\gamma}, 0\right)$.

${ }^{28}$ For brevity $\mu_{U}$ and $\mu_{F}$ are used instead of $\mu_{U}(d, d)$ and $\mu_{F}(d, d)$. 
with $\mu_{F}(d, d)$ being described above. The solution yields ${ }^{29}$

$$
\begin{aligned}
\hat{\mu}_{U} & =\delta \Delta w \gamma \frac{1-\gamma d \tau \mu_{F}(d, d)}{c-\delta \Delta w \gamma^{2}} \\
& =\frac{\delta \Delta w \gamma\left(c-\gamma^{2} \Delta w d^{2} \delta^{2}\right)}{\left(\gamma^{2} \Delta w d^{2} \delta^{2}\left(\tau^{2}-1\right)+c\right)\left(c-\delta \Delta w \gamma^{2}\right)} .
\end{aligned}
$$

Inserting $\hat{\mu}_{U}$ and $\mu_{F}(d, d)$ into $U$ 's objective function gives:

$$
E U_{U}\left(\hat{\mu}_{U} ; 1, d\right)=w_{1}-\delta \Delta w+\frac{\delta \Delta w c\left(c-\gamma^{2} \Delta w d^{2} \delta^{2}\right)^{2}}{2\left(c-\delta \Delta w \gamma^{2}\right)\left(\gamma^{2} \Delta w d^{2} \delta^{2}\left(\tau^{2}-1\right)+c\right)^{2}}
$$

Hence, $U$ does not want to deviate from the doping candidate equilibrium if and only if $E U_{U}(d, d) \geq E U_{U}\left(\hat{\mu}_{U} ; 1, d\right)$ which can be simplified to condition (13).

Now we have to check, whether the favorite wants to deviate from $\left(d_{U}, d_{F}\right)=$ $(d, d)$. Given $d_{U}=d$ and $\mu_{U}=\mu_{U}(d, d)$, in case of deviation $F$ would choose $d_{F}=1$ and that value of $\mu_{F}$ that maximizes his expected utility in the given situation. Let $\hat{\mu}_{F}$ denote this investment level. Unfortunately, it is not clear whether $\hat{\mu}_{F}$ corresponds to a situation where $d \mu_{U}(d, d)-\tau \hat{\mu}_{F}$ is located at the LHS or the RHS of the probability distribution. Hence, we have to check both cases.

Case 1: deviation still leads to the LHS

In this case, optimal deviation requires

$$
\begin{aligned}
\hat{\mu}_{F}^{L H S}= & \underset{\mu_{F}}{\arg \max } w_{1}-\frac{c}{2} \mu_{F}^{2} \\
& -\delta \Delta w\left(\gamma\left(d \mu_{U}(d, d)-\tau \mu_{F}\right)+\frac{\gamma^{2}}{2}\left(d \mu_{U}(d, d)-\tau \mu_{F}\right)^{2}+\frac{1}{2}\right)
\end{aligned}
$$

\footnotetext{
${ }^{29}$ Note that $\hat{\mu}_{U}-d \tau \mu_{F}(d, d)>-\frac{1}{\gamma}$ is satisfied. Furthermore, condition $\hat{\mu}_{U}<$ $d \tau \mu_{F}(d, d) \Leftrightarrow \frac{\left(c-\gamma^{2} \Delta w d^{2} \delta^{2}\right)}{\left(c-\gamma^{2} \Delta w \delta\right)}<d^{2} \tau^{2} \delta$ is also satisfied since the left-hand side is smaller and the right-hand side is larger than one due to (4).
} 
(with $\mu_{U}(d, d)$ being described above) leading to

$$
\begin{aligned}
\hat{\mu}_{F}^{L H S} & =\delta \Delta w \gamma \tau \frac{1+\gamma d \mu_{U}(d, d)}{\delta \Delta w \gamma^{2} \tau^{2}+c} \\
& =\frac{\delta \Delta w \gamma \tau\left(\gamma^{2} \Delta w d^{2} \delta^{2} \tau^{2}+c\right)}{\left(\gamma^{2} \Delta w d^{2} \delta^{2}\left(\tau^{2}-1\right)+c\right)\left(\delta \Delta w \gamma^{2} \tau^{2}+c\right)}
\end{aligned}
$$

and

$$
E U_{F}\left(\hat{\mu}_{F}^{L H S} ; d, 1\right)=w_{1}-\frac{\left(\gamma^{2} \Delta w d^{2} \delta^{2} \tau^{2}+c\right)^{2} c \delta \Delta w}{2\left(\delta \Delta w \gamma^{2} \tau^{2}+c\right)\left(\gamma^{2} \Delta w d^{2} \delta^{2}\left(\tau^{2}-1\right)+c\right)^{2}}
$$

Note that for this solution the LHS condition $d \mu_{U}(d, d)<\tau \hat{\mu}_{F}^{L H S}$ must hold, ${ }^{30}$ which can be rearranged to

$$
c\left(d^{2} \delta-\tau^{2}\right)<d^{2} \Delta w \tau^{2} \gamma^{2} \delta^{2}\left(\tau^{2}-1\right)
$$

Case 2: deviation leads to the RHS

Now optimal deviation requires

$$
\begin{aligned}
\hat{\mu}_{F}^{R H S}= & \underset{\mu_{F}}{\arg \max } w_{1}-\frac{c}{2} \mu_{F}^{2} \\
& -\delta \Delta w\left(\gamma\left(d \mu_{U}(d, d)-\tau \mu_{F}\right)-\frac{\gamma^{2}}{2}\left(d \mu_{U}(d, d)-\tau \mu_{F}\right)^{2}+\frac{1}{2}\right)
\end{aligned}
$$

yielding

$$
\begin{aligned}
\hat{\mu}_{F}^{R H S} & =\frac{\Delta w \tau \gamma \delta\left(1-d \gamma \mu_{U}(d, d)\right)}{c-\Delta w \tau^{2} \gamma^{2} \delta} \\
& =\frac{\Delta w \tau \gamma \delta\left(d^{2} \Delta w \gamma^{2} \delta^{2}\left(\tau^{2}-2\right)+c\right)}{\left(c-\Delta w \tau^{2} \gamma^{2} \delta\right)\left(d^{2} \Delta w \gamma^{2} \delta^{2}\left(\tau^{2}-1\right)+c\right)} .
\end{aligned}
$$

${ }^{30}$ However, the condition $d \mu_{U}(d, d)-\tau \hat{\mu}_{F}^{L H S} \geq-\frac{1}{\gamma}$ is always satisfied. 
and

$$
\begin{aligned}
E U_{F}\left(\hat{\mu}_{F}^{R H S} ; d, 1\right)= & \delta w_{2}+w_{1}(1-\delta) \\
& +\frac{c \Delta w \delta\left(d^{2} \Delta w \gamma^{2} \delta^{2}\left(\tau^{2}-2\right)+c\right)^{2}}{2\left(c-\Delta w \tau^{2} \gamma^{2} \delta\right)\left(d^{2} \Delta w \gamma^{2} \delta^{2}\left(\tau^{2}-1\right)+c\right)^{2}} .
\end{aligned}
$$

For this solution, the RHS condition

$$
\begin{aligned}
d \mu_{U}(d, d) & >\tau \hat{\mu}_{F}^{R H S} \Leftrightarrow \\
c\left(d^{2} \delta-\tau^{2}\right) & >d^{2} \Delta w \tau^{2} \gamma^{2} \delta^{2}\left(\tau^{2}-1\right)
\end{aligned}
$$

must hold, ${ }^{31}$ which is just the opposite of (A3).

Note that we must have that

$$
\begin{aligned}
\hat{\mu}_{F}^{L H S} & >\hat{\mu}_{F}^{R H S} \Leftrightarrow \\
c\left(d^{2} \delta-\tau^{2}\right) & >d^{2} \Delta w \tau^{2} \gamma^{2} \delta^{2}\left(\tau^{2}-1\right) .
\end{aligned}
$$

Since this condition contradicts (A3), only case 2 has to be considered in the following. ${ }^{32}$

Finally, the favorite does not want to deviate from the doping candidate equilibrium if and only if $E U_{F}(d, d) \geq E U_{F}\left(\hat{\mu}_{F}^{R H S} ; d, 1\right)$ which can be simplified to condition (14).

\footnotetext{
${ }^{31}$ Note that condition $d \mu_{U}(d, d)-\tau \hat{\mu}_{F}^{R H S}<\frac{1}{\gamma}$ is always satisfied.

${ }^{32}$ Moreover, note that $E U_{F}\left(\hat{\mu}_{F}^{R H S} ; d, 1\right)>E U_{F}\left(\hat{\mu}_{F}^{L H S} ; d, 1\right)$ holds.
} 


\section{References}

Baik, K.H. (1994), Effort levels in contests with two asymmetric players, Southern Economic Journal 61, 367-378.

Berentsen, A. (2002), The economics of doping, European Journal of Political Economy 18, 109-127.

Berentsen, A., Bruegger, E. and S. Loertscher (2004), The evolution of cheating in asymmetric contests, Discussion Paper.

Berentsen, A. and Y. Lengwiler (2004), Fraudulent accounting and other doping games, Journal of Institutional and Theoretical Economics 160, $402-415$.

Bird, E.J. and G.G. Wagner (1997), Sport as a common property resource, Journal of Conflict Resolution 41, 749-766.

Chen, K.-P. (2003), Sabotage in promotion tournaments, Journal of Law, Economics, and Organization 19, 119-140.

Drago, R., Garvey T.G. and G.K. Turnbull (1996), A collective tournament, Economics Letters 50, 223-227.

Eber, N. and J. Thépot (1999), Doping in sport and competition design, Louvain Economic Review 65, 435-446.

Epstein, G.S. and C. Hefeker (2003), Lobbying contests with alternative instruments, Economics of Governance 4, 81-89

Fairburn, J.A. and J.M. Malcomson (1994), Rewarding performance by promotion to a different job, European Economic Review 38, 683-690. 
Falconieri, S., Palomino, F. and J. Sákovics (2004), Collective versus individual sale of television rights in league sports, Journal of the European Economic Association 2, 833-862.

Fort, R. and J. Quirk (1995), Cross-subsidization, incentives, and outcomes in professional team sports leagues, Journal of Economic Literature 33, $1265-1299$.

Haugen, K.K. (2004), The performance-enhancing drug game, Journal of Sports Economics 5, 67-86.

Kesenne, S. (2005), Revenue sharing and competitive balance, Journal of Sports Economics 6, 98-106.

Konrad, K.A. (2000), Sabotage in rent-seeking contests, Journal of Law, Economics, and Organization 16, 155-165.

Konrad, K.A. (2005), Tournaments and multiple productive inputs: the case of performance enhancing drugs, IZA Discussion Paper No. 1844.

Kräkel, M. (2000), Relative deprivation in rank-order tournaments, Labour Economics 7, 385-407.

Kräkel, M. (2005), Helping and sabotaging in tournaments, International Game Theory Review 7, 211-228.

Lazear, E. P. (1989), Pay equality and industrial politics, Journal of Political Economy 97, 561-580.

Lazear, E.P. and S. Rosen (1981), Rank order tournaments as optimum labor contracts, Journal of Political Economy 89, 841-864. 
Maennig, W. (2002), On the economics of doping and corruption in international sports, Journal of Sports Economics 3, 61-89.

Martinson, B.C., Anderson, M.S. and R. de Vries (2005), Scientists behaving badly, Nature 435, 737-738.

Nalebuff, B.J. and J.E. Stiglitz (1983), Prizes and incentives: towards a general theory of compensation and competition, Bell Journal of Economics 3, 21-43.

Nti, K.O. (1999), Rent-seeking with asymmetric valuations, Public Choice $98,415-430$.

Preston, I. and S. Szymanski (2003), Cheating in contests, Oxford Review of Economic Policy 19, 612-624.

Szymanski, S. (2003), The economic design of sporting contests, Journal of Economic Literature 41, 1137-1187.

Szymanski, S. and S. Kesenne (2004), Competitive balance and gate revenue sharing in team sports, Journal of Industrial Economics 52, 165-177.

Tirole, J. (1996), A theory of collective reputation (with applications to the persistence of corruption and to firm quality), Review of Economic Studies 63, 1-22. 